\title{
26 Research Square \\ miR-188 promotes Oxaliplatin resistances through targeting RASA1 in colon cancer cells
}

\section{Xijia Zhu}

the second affiliated hospital of Guilin medical hospital

\section{Xishun Luo}

the second affiliated hospital of Guilin medical hospital

\section{Zhike Song}

the second affiliated hospital of Guilin medical hospital

\section{Shiyu Jiang}

the second affilated hospital of Guilin meidical hospital

\section{Xiangkai Long}

the second affiliated hospital of Guilin medical hospital

\section{Xuanyuan Gao}

Guilin medical college

\section{Xinyang Xie}

Guilin Medical college

\section{Laijian Zheng}

Guilin Medical College

Haipeng Wang ( $\nabla$ wanghaipeng201605@163.com )

the second affiliated hospital of Guilin medical university

\section{Research article}

Keywords: chemoresistance, miR-188, colon cancer, oxaliplatin, RASA1, apoptosis

Posted Date: December 4th, 2019

DOl: https://doi.org/10.21203/rs.2.18041/v1

License: (c) (i) This work is licensed under a Creative Commons Attribution 4.0 International License.

Read Full License 


\section{Abstract}

Background: The chemoresistance of colon cancer cells limits the efficacy of chemotherapy. miR-188 has been shown to be down-regulated in various types of cancer. The aim of this study was to explore the molecular mechanism of miR-188 in drug resistant cancer cells.

Materials and methods: we examined the effects of miR-188 on the sensitivity of colon cancer cells to oxaliplatin (OXA) by using SW480/OXA cell line. The target of miR-188 was determined by luciferase activity assay. The cell cycle distribution were detected by flow cytometry. The expression of p21, Hoechst 33342 staining and Annexin $V$ assays were used to detect the cell apoptosis.

Results: The expression of miR-188 was significantly increased in SW480/OXA cells compared with SW480 cells. By luciferase assay we found that miR-188 miRNA binds to RASA1 (Ras GTPase-activating protein 1, also known as p120RasGAP), and overexpression of miR-188 inhibited RASA1 expression by binding to the 3'-untranslated region of RASA1 mRNA. In addition, suppression of miR-188 enhanced the chemosensitivity of the oxaliplatin-resistant colon cancer cells. Furthermore, suppression of RASA1 abrogated the increasement of cell apoptosis induced by miR-188 inhibitor, while, overexpression of RASA1 induced cell apoptosis in SW480/OXA cells. Our results suggested that miR-188 played chemoresistant role in colon cancer through regulating RASA1 expression.

Conclusion: The findings of our study suggest that target miR-188 is capable of enhancing the chemosensitivity of colon cancer cells by promoting RASA1.

\section{Background}

Colon cancer is the third most common cancer with high cancer-related deaths worldwide.

Chemoresistance is still a crucial challenge for the treatment of colon cancer. microRNAs (miRNAs) take part in complex physiological and pathological processes by suppression the expression of their target gene through binding to the $3^{\prime}$ untranslated region (UTR) [1]. Each miRNA has multiple targeted mRNAs, thus consequentially result in a variety of effects [2]. Recently, some studies have highlighted miRNAs linked to chemoresistant phenotype of different tumors, mainly through abnormal regulation of apoptosis [3], cell cycle distribution [4], and activity of drug efflux transporters [5]. Correction of these miRNAs using miRNA mimics or antagomirs can normalize the gene regulatory network and signaling pathways and sensitize cancerous cells to chemotherapy. Therefore, miRNA-based gene therapy provides an attractive anti-tumor approach for integrated cancer therapy.

miR-188 has been shown to be down-regulated in various types of cancer, such as cervical cancer [6], non-small cell lung cancer [7], gastric cancer[8], glioma [9], coloretal cancer [10], oral squamous cell carcinoma [11], hepatocellular carcinoma [12], prostate cancer [13], acute myeloid leukemia [14] and rectal cancer [15]. Previous studies showed that miR-188 expression was significantly down-regulated at the tumor sites and cancer cells [13]. In vitro transfection of miR-188 reduced cell proliferation and migration potential and promoted cell apoptosis [7]. In xenograft model, miR-188 inhibited tumor growth 
derived from cancer cells [7]. In colon cancer, $\mathrm{miR}-188-3 p$ has been shown as a novel independent prognostic factor in colorectal cancer patients, which can be partly explained by its effect on MLLT4 expression and migration of cancer cells [10]. More importantly, miR-188-5p was correlated with complete pathological response to neoadjuvant chemoradiotherapy in locally advanced rectal cancer [15]. However, the molecular mechanism of miR-188 in drug resistant cancer cells have not been found.

RASA1 (Ras GTPase-activating protein 1, also known as p120RasGAP), is the first identified RasGAP protein. RASA1 has been involved in many biological processes including actin filament polymerization, cell apoptosis and migration [16]. RASA1 has also been proven to be a cancer suppressor gene in many types of cancer [17-25]. RASA1 suppresses the actions of RAS by enhancing the weak intrinsic GTPase activity of RAS proteins, leading to an increase in the inactive GDP-bound form of RAS, which leads to aberrant intracellular signaling through the RAS-RAF-ERK pathway. Previous study showed that RASA1

protein levels were significantly decreased in colon cancer cells and RASA1 was a target gene of miR-21, promoted malignant behaviors of colon cancer cells [26]. RASA1 up-regulation inhibited cell proliferation and turned off the RAS signaling pathway [26]. Additionally, miR-223 and RASA1 demonstrated an inverse correlation in colorectal cancer (CRC) patients tissues [27] and RASA1 was validated as a target of miR-335 which was downregulation in CRC [28]. Besides, several studies indicated that oncomicroRNAs (micorRNA-21 and micorRNA-182) can promote tumor angiogenesis or lymph node metastasis by targeting RASA1 $[29,30]$.

Here, we shown that the expression of miR-188 was significantly increased in oxaliplatin (OXA) resistant colon cancer cells compared with colon cancer cells. To figure out the detail roles of miR-188 on colon cancer chemoresistance, we performed the bioinformatics analysis to show that RASA1 was a target of miR-188. We further examined the effects of miR-188 and its target gene RASA1 on chemoresistance of colon cancer.

\section{Methods}

\section{Cell culture and treatment}

Human colon cancer cell line SW480 and human embryonic kidney cell line 293T were purchased from ATCC. To induce OXA resistant SW480 cells, the cells were seeded onto 24 well plate before chemotherapeutics treatment. Next, SW480 cells were cultured in the presence of $2.5 \mathrm{mM}$ oxaliplatin (OXA) for 24 hours followed by three days in fresh medium without a drug. This procedure was repeated six times. Both of SW480 and SW480/OXA cells were cultured in Dulbecco Modified Eagle Medium (DMEM) with $10 \%$ fetal bovine serum (FBS) and $1 \%$ penicillin/streptomycin solution in a cell incubator at $37^{\circ} \mathrm{C}, 5 \% \mathrm{CO}_{2}$, with saturated humidity.

\section{Hoechst 33342 staining}


Cells were seeded onto fibronectin coated 12 well plate, after treatment, Hoechst staining solution (5 $\mathrm{mg} / \mathrm{ml}$ ) was added to cells and incubated for 10 mins at room temperature. Then cells were washed with PBS for three time. DAPI was used to stain the nuclear DNA. Images were collected by Nikon T1-SAM.

\section{Real-time PCR}

Total RNAs were isolated from cells using TRIzol (Invitrogen, USA) according to the manufacturer's instruction. First stand of cDNA was synthesized by the Reverse Transcription Kit (Applied Biosystems, USA). Real-Time PCR reaction was performed using SYBR Green PCR Master Mix (Applied Biosystems). $\mathrm{U} 6$ and GAPDH were used as internal references. The relative levels were calculated by the 2- $\triangle \triangle \mathrm{Ct}$ method. Primer sequences used in the study were: GAPDH: 5'TGTTCGTCATGGGTGTGAAC3' (forward) and 5'-ATGGCATGGACTGTGGTCAT3' (reverse), RASA1: 5'CAGTGGACGAAGGTGACTCT3' (forward)and 5'AGGCGTTCTTCTGCTATCGT3' (reverse), U6: 5' -CTCGCTTCGGCAGCACA3' (forward) and 5'AACGCTTCACGAATTTGCGT3' (reverse), All: 5'CTCAACTGGTGTCGTGGA3', has-miR-188: 5'CTCAACTGGTGTCGTGGAGTCGGCAATTCAGTTGAGCCCTCCAC3' (forward) and 5'ACACTCCAGCTGGGCATCCCTTGCATGGTGG3' (reverse).

\section{Luciferase activity assay}

Luciferase constructs (psiECHECK-2, Promega) were made by ligating oligonucleotides containing the wild type or mutant type of RASA1 3'UTR downstream of the luciferase gene. Cells were co-transfected with RASA1 (WT/MUT) and NC or miR188 mimics by Lipofectamine 2000 (Invitrogen). Luciferase activity was detected 48 hours after transfection by a luciferase reporter gene assay kit (Sigma). Experiments were performed in triplicate in three independent experiments.

\section{Cell transfection}

miR-188 inhibitor, mimics, RASA1 siRNA and RASA1 overexpressed plasmid were purchased from Shanghai GenePharma and transfected to cells by Lipofectamine 2000 according to manufacturer's instructions.

\section{Western blot}

The cells were harvested and disrupted in lysis buffer containing phosphorylase inhibitor cocktail (Roche) and phenylmethanesulfonyl fluoride (PMSF). An equal amount $(30 \mathrm{mg}$ ) of each protein sample was electrophoresed on an 8-12\% SDS-polyacrylamide gel and transferred onto polyvinylidene fluoride 
(PVDF) membranes. Subsequently, the membranes were incubated with specific antibodies after being blocked using $5 \%$ skimmed milk powder for $1 \mathrm{~h}$ at room temperature. The main primary antibodies included p21 (Abcam, ab227443, 1:1000), RASA1 (Abcam, ab2922, 1:1000), Ras-GTP (Abcam, ab69747, 1:2000) and GAPDH (Abcam, ab9485, 1:5000) were added to incubate at $4^{\circ} \mathrm{C}$ overnight. Goat anti-rabbit (1:5000) or goat anti-mouse (1:5000) IgG-HRP (Sigma) were added and incubated at room temperature for $1 \mathrm{~h}$. The relative expression of the target protein was divided by an internal control.

\section{Immunofluorescence assay}

Cells were seeded onto fibronectin coated 12 well plate, after treatment, cells were fixed by $4 \%$ paraformaldehyde at room temperature for 10 mins and block by $1 \%$ BSA in PBS. The slides were incubated with the primary antibody (RASA1, Abcam, ab2922) at room temperature for 1 hour and washed three times with PBS. Alexa Fluor 555 goat anti-mouse secondary antibody (Abcam, ab150114) was used. After incubation with secondary antibody at room temperature for 40 mins, the slides were washed with PBS for three times and DAPI was used to stain the DNA.

\section{Apoptosis assay}

Cell apoptosis was performed by flow cytometry. Using apoptosis Kit (Roche) according to manufacturer's instructions, double staining with FITC-Annexin $\mathrm{V}$ and propidium iodide (PI) of cells were performed and analyzed suing a FACScan ${ }^{\circledR}$ flow cytometer (BD, USA). Experiments were performed in triplicate.

\section{Cell cycle analysis}

Cells $\left(2 \times 10^{5} /\right.$ well $)$ were seeded onto 6 well plate. Twelve hours later, cells were transfected. Forty eight hours after transfection, cells were harvested, fixed in $1 \%$ paraformaldehyde, washed with PBS, and stained with $5 \mathrm{mg} / \mathrm{ml}$ propidium iodide (PI) in PBS supplemented with RNase A (Roche) for 30 mins at room temperature. The cells were analyzed using FACS Calibur flow cytometer. One parameter histogram was plotted according to the distribution of nuclear DNA content in each cell detected by flow cytometer. Cells in each individual phase of the cell cycle were determined based on their DNA ploidy profile.

\section{Statistical analysis}

The statistical analysis was performed using SPSS 15.0 (SPSS, USA) with unpaired two-tailed student's t test. For multivariate groups comparison, ANOVO was performed. ${ }^{*} P<0.05, * * P<0.01, * \star \star x<0.001$. 


\section{Results}

\section{Inhibition of miR-188 up-regulates RASA1 expression.}

To assess the association of miR-188 expression in OXA resistant SW480 cells, we performed real-time PCR on SW480 and SW480/OXA cells. As shown in Figure 1A, the expression of miR-188 was significantly upregulated in SW480/OXA cells as compared with SW480 cells, suggesting that the expression of miR-188 was related to drug resistant of colon cancer.

To further dissect the function of miR-188, we predicted miR-188 potential targeting genes in Targetscan and miRDB database. RASA1 was among the most potential miR-188 targets in the prediction. We found that the mRNA expression of RASA1 was reduced in SW480/OXA cells (Figure 1B). To confirm whether RASA1 was a direct target of miR-188, we generated luciferase reporter constructs with the 3 ' UTR of RASA1 mRNA and transfected them to 293T cells with miR-188 mimics (Figure 1C). We found that cotransfected with miR-188 decreased luciferase activity of wild type form of RASA1, while mutation of the binding sites did not shown significant changes (Figure 1C).

In order to detect the regulatory effects of miR-188 on RASA1 expression, SW480 or SW480/OXA cells were transfected with miR-188 mimics or inhibitor, we found that miR-188 mimics induced miR-188 expression, and reduced RASA1 expression, while miR-188 inhibitor reduced miR-188 expression and increased RASA1 expression (Figure 1D-E). We also detected the expression of RASA1 by immunofluorescence, and the results shown that miR-188 regulated the protein expression of RASA1 (Figure 1F). Our data shown that inhibition of miR-188 could increase the expression of its target gene RASA1 in SW480 and SW480/OXA cells.

\section{Targeting miR-188 induces cell apoptosis in SW480/OXA cells.}

To further detect the role of miR-188 on the apoptosis of colon cancer cells, we transfected miR-188 mimics or inhibitor to SW480 and SW480/OXA cells, the results shown that miR-188 mimics significantly decreased the expression of RASA1 and miR-188 inhibitor significantly increased the expression of RASA1 (Figure 2A). In addition, we detected the expression of the apoptosis related gene p21, and we found that the expression of p21 was decreased in miR-188 mimics transfected cells and increased in miR-188 inhibitor transfected cells (Figure 2A). More importantly, comparing with SW480 cells, the expression of p21 was lower in miR-188 mimics transfected SW480/OXA cells (Figure 2A). As RASA1 suppresses the actions of RAS by enhancing the hydrolysis of weak intrinsic GTPase activity of RAS proteins into an inactive GDP-bound form of RAS, we found that the expression of Ras-GTP was changed following by miR-188 mimics or inhibitor transfection (Figure 2A). Our results suggested that targeted 
miR-188 induced cell apoptosis of OXA resistant colon cancer cells (Figure 2A). Moreover, both the Hoechst 33342 staining and Annexin $V$ assay showed that miR-188 mimics inhibited cell apoptosis and miR-188 inhibitor promoted cell apoptosis in SW480 and SW480/OXA cellsand (Figure 2B and C). Next, we determined the role of miR-188 on cell cycle progression, SW480 or SW480/OXA cells were transfected with negative control, miR-188 mimics or inhibitor. As shown in Figure 2D, miR-188 mimics reduced the $G_{1}$ cell population and induced $S$ phase percentage, while the $G_{1}$ cell population was bigger in miR-188 inhibitor transfected cells than that in negative control cells, and the $S$ phase percentage was smaller in miR-188 inhibitor transfected cells (Figure 2D). Our results suggesting that targeting miR-188 sensitized OXA resistant SW480 cells.

\section{Overexpression of RASA1 accelerates cell apoptosis and delays cell cycle.}

As a direct target of miR-188, we next determined the effect of RASA1 on colon cancer cells or OXA resistant colon cancer cell apoptosis and cell cycle progression. We transiently transfected RASA1 overexpressed plasmid or siRNA and the results shown that overexpression of RASA1 significantly increased RASA1, while RASA1 siRNA significantly decreased RASA1 expression in both SW480 and SW480/OXA cells (Figure 3A-B). The expression of the apoptosis related gene p21 enhanced significantly in RASA1 overexpressed cells and reduced in siRASA1 transfected cells (Figure 3B). We also shown that overexpression of RASA1 suppressed Ras-GTP and siRASA1 induced Ras-GTP in both SW480 and SW480/OXA cells (Figure 3B). In addition, we found that RASA1 promotes cell apoptosis both in SW480 and SW480/OXA cells by Hoechst 33342 staining and Annexin $V$ assays (Figure 3C-D) and overexpression of RASA1 induced the expression of p21 (Figure 3B). Conversely, suppression of RASA1 by siRNA decreased the expression of p21 (Figure 3B) and reduced cell apoptosis (Figure 3C-D). We also measured the cell cycle distribution in overexpression of RASA1 or suppression of RASA1 cells. Overexpression of RASA1 accumulated $\mathrm{G}_{1}$ phase cells and reduced $S$ phase cell population in both SW480 and SW480/OXA cells (Figure 3E), while suppression of RASA1 decreased $\mathrm{G}_{1}$ percentage and increased $S$ phase percentage (Figure 3E). These findings further confirmed that RASA1 induced cell apoptosis of OXA resistant colon cancer cells.

\section{Suppression of miR-188 promotes SW480/OXA cell apoptosis via increasing RASA1 expression.}

We next confirmed whether RASA1 could affect the effects of miR-188 on cell apoptosis and cell cycle distribution. RASA1 and p21 was increased in miR-188 mimics induced SW480 cells by transfected with RASA1 overexpressed plasmid and RASA1 and p21 was inhibited in miR-188 inihibitor induced SW480/OXA cells by transfected with siRASA1 (Figure 4A). Additionally, the expression of Ras-GTP did the reverse (Figure 4A). Hoechst 33342 staining and Annexin $V$ assays showed that overexpression of RASA1 enhanced cell apoptosis in miR-188 mimics induced SW480 cells while suppression of RASA1 abrogated the increasement of SW480/OXA cells apoptosis induced by miR-188 inhibitor (Figure 4B-C). 
Moreover, RASA 1 increased $\mathrm{G}_{1}$ phase and decreased $\mathrm{S}$ phase of cell cycle in miR-188 mimics induced SW480 cells (Figure 4D), while suppression of RASA1 decreased $G_{1}$ phase and increased $S$ phase of cell cycle in miR-188 inhibitor induced SW480/OXA cells (Figure 4D). These results suggested that targeted miR-188 induced SW480/OXA cell apoptosis via enhancing RASA1 expression.

\section{Discussion}

In this study, we found that miR-188 was significantly up-regulated in SW480/OXA cells. miR-188 mimics caused a significant inhibition of OXA-induced cell apoptosis, while blocking miR-188 increased OXA resistant colon cancer cell apoptosis significantly. Additionally, we found that miR-188 was involved in repression of RASA1 expression through regulating its transcription. Moreover, overexpression of miR-188 conferred OXA resistance, while down-regulation of miR-188 sensitized resistant cells to OXA, which was blocked by inhibition of RASA1. Our findings may provide new therapeutic target for colon cancer cell resistance to OXA.

Mounting evidences implicate an important role of miR-188 in cancer development. However, little is known about its expression pattern in OXA resistant colon cancer cells. Here, we shown that the expression of miR-188 was significantly increased in OXA resistant colon cancer cells. Previous genomewide miRNA sequencing data of 228 colorectal cancer patients showed that six miRNAs, including miR$188-3 p$ were identified as strong predictor of patient survival. miR-188-3p promotes colorectal cancer cell migration both in vitro and in vivo partly through regulation of MLLT4 expression and high miR-188$3 p$ expression proved to be an independent prognostic factor [10]. Additionally, a previously published study reported an involvement miR-188-5p in rectal cancer and response to neoadjuvant radiochemotherapy [15]. Our results were partly consistent with previous studies showing that miR-188 promoted colon cancer progression. In the present study, we found that down-regulation of miR-188 promoted cell apoptosis and up-regulation of miR-188 inhibited cell apoptosis in SW480/OXA cells, suggesting that targeting miR-188 (miR-188 inhibitor) induced cell apoptosis of OXA resistant cells. Moreover, suppression of miR-188 induced $G_{1}$ phase cell cycle arrest and increased cell apoptosis. Our results were consistent with previous study showing that miR-188 controlled cell cycle progression via down-regulation of multiple cyclin/CDK complexes involved in $\mathrm{G}_{1} / \mathrm{S}$ transition $[31,13]$. Although miR188 served as a tumor suppressor and was down-regulated in multiple types of cancer, we found that miR-188 was up-regulated in OXA resistant colon cancer and targeting miR-188 induced cell apoptosis in OXA resistant cells.

Of many potential target genes for miR-188 predicted by TargetScan and miRDB Human database, RASA1 was selected for the percent study. RASA1 acts as a suppressor of RAS function and enhances the weak intrinsic GTPase activity of RAS proteins resulting in the inactive GDP-bound form of RAS, thus involving in many biological processes [16]. Previous studies showed that RASA1 played as a tumor suppressor in hepatocellular carcinoma [32], human bronchial epithelial cells [33], breast cancer [34] and melanoma [35]. Recently, RASA1 was found to be significantly decreased in colon cancer cells and RASA1 was a target gene of miR-21, miR-223 and miR-335 [26] [27] [28]. Moreover, onco-microRNAs 
(micorRNA-21 and micorRNA-182) promote tumor angiogenesis or lymph node metastasis by targeting RASA1 [29, 30]. p21, a tumor suppressive protein, is a negative regulator in the $G_{1} / S$ transition [36] and down-regulation of p21 is involved in various human cancers [37]. Here, we showed that there was a negative correlation between miR-188 and RASA1 in OXA resistant colon cancer cells. Overexpression of RASA1 accelerated cell apoptosis, induced the expression of p21 and delayed cell cycle progression. More importantly, miR-188 mimics reduced cell apoptosis in SW480 cells, while overexpression of RASA1 enhanced cell apoptosis in miR-188 mimics transfected SW480 cells. In contrast, suppression of RASA1 abrogated cell apoptosis in miR-188 inhibitor transfected SW480/OXA cells. These results suggested that targeted miR-188 induced SW480/OXA cell apoptosis via enhancing RASA1 expression.

\section{Conclusion}

In conclusion, the findings of our study suggested that inhibition of miR-188 was capable of enhancing the chemosensitivity of colon cancer cells by promoting RASA1, which provided new insights into the treatment of OXA resistant colon cancer. Our study provided a basis for new targeted therapy in colon cancer. This is especially important in view of drug resistance observed with colon cancer targeted drug oxaliplatin, which is widely used clinically.

\section{Abbreviations}

CRC:colorectal cancer

OXA:oxaliplatin

RASA1:Ras GTPase-activating protein 1

UTR: untranslated region

\section{Declarations}

\section{Ethics approval and consent to participate}

Before tissue and cells collection, all patients were administered the informed consent and this study was approved by the Research Ethics Committee of the Second Affiliated Hospital of Guilin Medical University.Written informed consent was obtained from the participants.

\section{Consent to publish}

Not applicable

\section{Availability of data and materials}


The data used in this research are available from the corresponding author on reasonable request.

\section{Competing Interests}

The authors declare that they have no conflict of interest.

\section{Funding}

This study was supported by the Guilin Scientific Research and Technology Development Plan Project (20170109-50).

\section{Author's contribution}

HW conceived and designed research, drafted and revised manuscript;XZ, XSL, ZS, SJ and XG performed experiments; XGL, XX and LZ analyzed data and prepared figures; All authors approved final version of manuscript; XZ and XSL edited andrevised manuscript.

\section{Acknowledgement}

Not applicable

\section{Reference}

1. Meister G, Tuschl T. Mechanisms of gene silencing by double-stranded RNA. Nature. 2004;431(7006):343-9. doi:10.1038/nature02873.

2. Garofalo $M$, Croce $C M$. microRNAs: Master regulators as potential therapeutics in cancer. Annual review of pharmacology and toxicology. 2011;51:25-43. doi:10.1146/annurev-pharmtox-010510100517.

3. Xie Y, Tobin LA, Camps J, Wangsa D, Yang J, Rao M et al. MicroRNA-24 regulates XIAP to reduce the apoptosis threshold in cancer cells. Oncogene. 2013;32(19):2442-51. doi:10.1038/onc.2012.258.

4. Yamanaka S, Campbell NR, An F, Kuo SC, Potter JJ, Mezey E et al. Coordinated effects of microRNA494 induce $G(2) / M$ arrest in human cholangiocarcinoma. Cell cycle. 2012;11(14):2729-38. doi:10.4161/cc. 21105 .

5. Zhu H, Wu H, Liu X, Evans BR, Medina DJ, Liu CG et al. Role of MicroRNA miR-27a and miR-451 in the regulation of MDR1/P-glycoprotein expression in human cancer cells. Biochemical pharmacology. 2008;76(5):582-8. doi:10.1016/j.bcp.2008.06.007.

6. Gao C, Zhou C, Zhuang J, Liu L, Liu C, Li H et al. MicroRNA expression in cervical cancer: Novel diagnostic and prognostic biomarkers. Journal of cellular biochemistry. 2018;119(8):7080-90. doi:10.1002/jcb.27029. 
7. Zhao L, Ni X, Zhao L, Zhang Y, Jin D, Yin W et al. MiroRNA-188 Acts as Tumor Suppressor in NonSmall-Cell Lung Cancer by Targeting MAP3K3. Molecular pharmaceutics. 2018;15(4):1682-9. doi:10.1021/acs.molpharmaceut.8b00071.

8. Peng Y, Shen X, Jiang H, Chen Z, Wu J, Zhu Y et al. miR-188-5p Suppresses Gastric Cancer Cell Proliferation and Invasion via Targeting ZFP91. Oncology research. 2018;27(1):65-71. doi:10.3727/096504018X15191223015016.

9. Li N, Shi H, Zhang L, Li X, Gao L, Zhang G et al. miR-188 Inhibits Glioma Cell Proliferation and Cell Cycle Progression Through Targeting beta-Catenin. Oncology research. 2018;26(5):785-94. doi:10.3727/096504017X15127309628257.

10. Pichler M, Stiegelbauer V, Vychytilova-Faltejskova P, Ivan C, Ling H, Winter E et al. Genome-Wide miRNA Analysis Identifies miR-188-3p as a Novel Prognostic Marker and Molecular Factor Involved in Colorectal Carcinogenesis. Clinical cancer research: an official journal of the American Association for Cancer Research. 2017;23(5):1323-33. doi:10.1158/1078-0432.CCR-16-0497.

11. Wang L, Liu H. microRNA-188 is downregulated in oral squamous cell carcinoma and inhibits proliferation and invasion by targeting SIX1. Tumour biology: the journal of the International Society for Oncodevelopmental Biology and Medicine. 2016;37(3):4105-13. doi:10.1007/s13277-0154246-9.

12. Fang $F$, Chang RM, Yu L, Lei $X$, Xiao S, Yang H et al. MicroRNA-188-5p suppresses tumor cell proliferation and metastasis by directly targeting FGF5 in hepatocellular carcinoma. Journal of hepatology. 2015;63(4):874-85. doi:10.1016/j.jhep.2015.05.008.

13. Zhang H, Qi S, Zhang T, Wang A, Liu R, Guo J et al. miR-188-5p inhibits tumour growth and metastasis in prostate cancer by repressing LAPTM4B expression. Oncotarget. 2015;6(8):6092-104. doi:10.18632/oncotarget.3341.

14. Jinlong $S$, Lin F, Yonghui L, Li Y, Weidong W. Identification of let-7a-2-3p or/and miR-188-5p as prognostic biomarkers in cytogenetically normal acute myeloid leukemia. PloS one. 2015;10(2):e0118099. doi:10.1371/journal.pone.0118099.

15. Della Vittoria Scarpati G, Falcetta F, Carlomagno C, Ubezio P, Marchini S, De Stefano A et al. A specific miRNA signature correlates with complete pathological response to neoadjuvant chemoradiotherapy in locally advanced rectal cancer. International journal of radiation oncology, biology, physics. 2012;83(4):1113-9. doi:10.1016/j.jirobp.2011.09.030.

16. Pamonsinlapatham P, Hadj-Slimane R, Lepelletier Y, Allain B, Toccafondi M, Garbay C et al. p120-Ras GTPase activating protein (RasGAP): a multi-interacting protein in downstream signaling. Biochimie. 2009;91(3):320-8. doi:10.1016/j.biochi.2008.10.010.

17. Yang XY, Guan M, Vigil D, Der CJ, Lowy DR, Popescu NC. p120Ras-GAP binds the DLC1 Rho-GAP tumor suppressor protein and inhibits its RhoA GTPase and growth-suppressing activities. Oncogene. 2009;28(11):1401-9. doi:10.1038/onc.2008.498.

18. Calvisi DF, Ladu S, Conner EA, Seo D, Hsieh JT, Factor VM et al. Inactivation of Ras GTPaseactivating proteins promotes unrestrained activity of wild-type Ras in human liver cancer. Journal of 
hepatology. 2011;54(2):311-9. doi:10.1016/j.jhep.2010.06.036.

19. Mai A, Veltel S, Pellinen T, Padzik A, Coffey E, Marjomaki V et al. Competitive binding of Rab21 and p120RasGAP to integrins regulates receptor traffic and migration. The Journal of cell biology. 2011;194(2):291-306. doi:10.1083/jcb.201012126.

20. Sun D, Yu F, Ma Y, Zhao R, Chen X, Zhu J et al. MicroRNA-31 activates the RAS pathway and functions as an oncogenic MicroRNA in human colorectal cancer by repressing RAS p21 GTPase activating protein 1 (RASA1). The Journal of biological chemistry. 2013;288(13):9508-18. doi:10.1074/jbc.M112.367763.

21. Anand S, Majeti BK, Acevedo LM, Murphy EA, Mukthavaram R, Scheppke L et al. MicroRNA-132mediated loss of p120RasGAP activates the endothelium to facilitate pathological angiogenesis. Nature medicine. 2010;16(8):909-14. doi:10.1038/nm.2186.

22. Organ SL, Hai J, Radulovich N, Marshall CB, Leung L, Sasazuki T et al. p120RasGAP is a mediator of rho pathway activation and tumorigenicity in the DLD1 colorectal cancer cell line. PloS one. 2014;9(1):e86103. doi:10.1371/journal.pone.0086103.

23. Sharma SB, Lin CC, Farrugia MK, McLaughlin SL, Ellis EJ, Brundage KM et al. MicroRNAs 206 and 21 cooperate to promote RAS-extracellular signal-regulated kinase signaling by suppressing the translation of RASA1 and SPRED1. Molecular and cellular biology. 2014;34(22):4143-64. doi:10.1128/MCB.00480-14.

24. Liu Y, Liu T, Sun Q, Niu M, Jiang Y, Pang D. Downregulation of Ras GTPaseactivating protein 1 is associated with poor survival of breast invasive ductal carcinoma patients. Oncology reports. 2015;33(1):119-24. doi:10.3892/or.2014.3604.

25. Li Z, Li D, Zhang G, Xiong J, Jie Z, Cheng $\mathrm{H}$ et al. Methylation-associated silencing of MicroRNA-335 contributes tumor cell invasion and migration by interacting with RASA1 in gastric cancer. American journal of cancer research. 2014;4(6):648-62.

26. Gong B, Liu WW, Nie WJ, Li DF, Xie ZJ, Liu C et al. MiR-21/RASA1 axis affects malignancy of colon cancer cells via RAS pathways. World journal of gastroenterology. 2015;21(5):1488-97. doi:10.3748/wjg.v21.i5.1488.

27. Sun D, Wang C, Long S, Ma Y, Guo Y, Huang Z et al. C/EBP-beta-activated microRNA-223 promotes tumour growth through targeting RASA1 in human colorectal cancer. British journal of cancer. 2015;112(9):1491-500. doi:10.1038/bjc.2015.107.

28. Lu Y, Yang H, Yuan L, Liu G, Zhang C, Hong M et al. Overexpression of miR-335 confers cell proliferation and tumour growth to colorectal carcinoma cells. Molecular and cellular biochemistry. 2016;412(1-2):235-45. doi:10.1007/s11010-015-2630-9.

29. Zhang L, Zhan X, Yan D, Wang Z. Circulating MicroRNA-21 Is Involved in Lymph Node Metastasis in Cervical Cancer by Targeting RASA1. International journal of gynecological cancer: official journal of the International Gynecological Cancer Society. 2016;26(5):810-6. doi:10.1097/IGC.0000000000000694. 
30. Zhu YJ, Xu B, Xia W. Hsa-mir-182 downregulates RASA1 and suppresses lung squamous cell carcinoma cell proliferation. Clinical laboratory. 2014;60(1):155-9.

31. Wu J, Lv Q, He J, Zhang H, Mei X, Cui K et al. MicroRNA-188 suppresses G1/S transition by targeting multiple cyclin/CDK complexes. Cell communication and signaling: CCS. 2014;12:66. doi:10.1186/s12964-014-0066-6.

32. Chen YL, Huang WC, Yao HL, Chen PM, Lin PY, Feng FY et al. Down-regulation of RASA1 Is Associated with Poor Prognosis in Human Hepatocellular Carcinoma. Anticancer research. 2017;37(2):781-5. doi:10.21873/anticanres.11377.

33. Hayashi T, Desmeules P, Smith RS, Drilon A, Somwar R, Ladanyi M. RASA1 and NF1 are Preferentially Co-Mutated and Define A Distinct Genetic Subset of Smoking-Associated Non-Small Cell Lung Carcinomas Sensitive to MEK Inhibition. Clinical cancer research: an official journal of the American Association for Cancer Research. 2018;24(6):1436-47. doi:10.1158/1078-0432.CCR-17-2343.

34. Suarez-Cabrera C, Quintana RM, Bravo A, Casanova ML, Page A, Alameda JP et al. A Transposonbased Analysis Reveals RASA1 Is Involved in Triple-Negative Breast Cancer. Cancer research. 2017;77(6):1357-68. doi:10.1158/0008-5472.CAN-16-1586.

35. Sung $\mathrm{H}$, Kanchi KL, Wang X, Hill KS, Messina JL, Lee JH et al. Inactivation of RASA1 promotes melanoma tumorigenesis via R-Ras activation. Oncotarget. 2016;7(17):23885-96. doi:10.18632/oncotarget.8127.

36. Pines J, Rieder CL. Re-staging mitosis: a contemporary view of mitotic progression. Nature cell biology. 2001;3(1):E3-6. doi:10.1038/35050676.

37. Cai K, Dynlacht BD. Activity and nature of p21(WAF1) complexes during the cell cycle. Proceedings of the National Academy of Sciences of the United States of America. 1998;95(21):12254-9. doi:10.1073/pnas.95.21.12254.

\section{Figures}


A

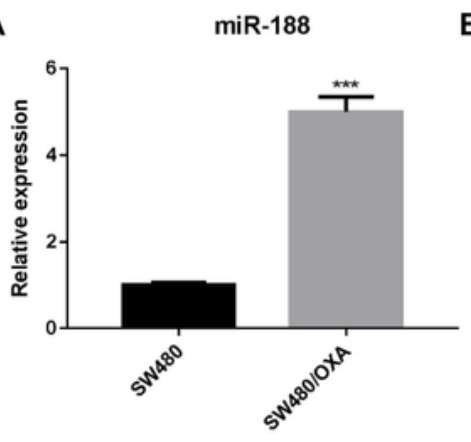

miR-188

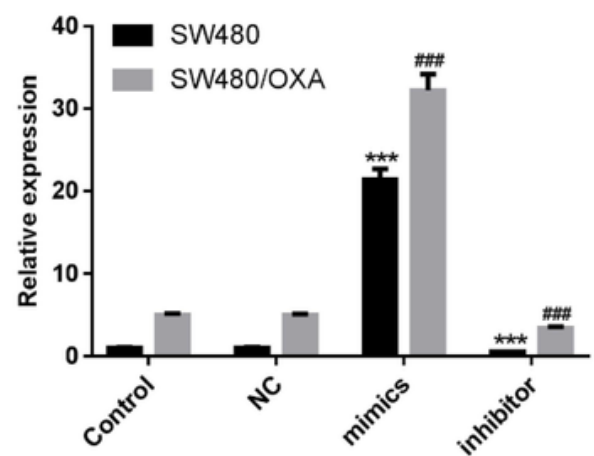

F

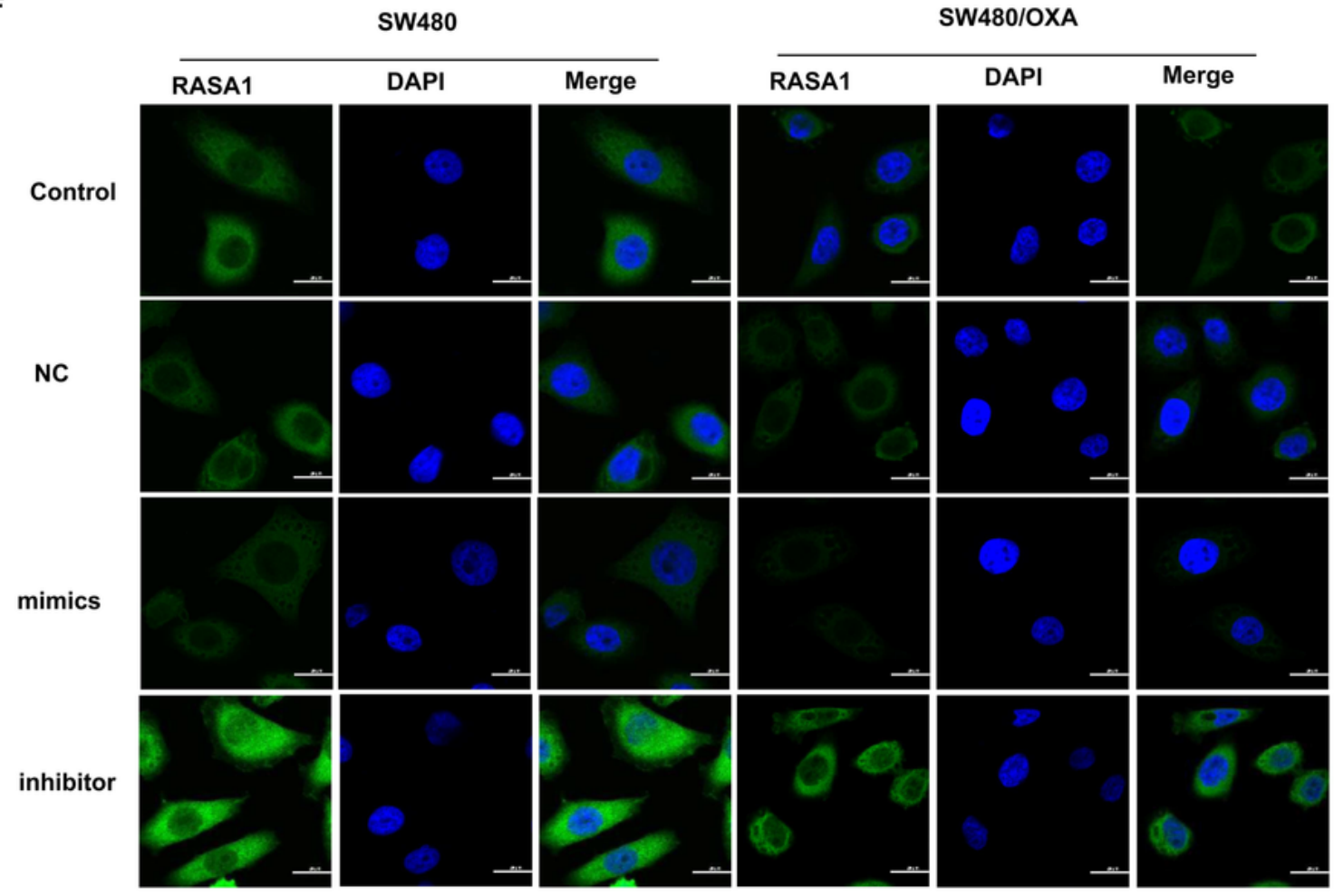

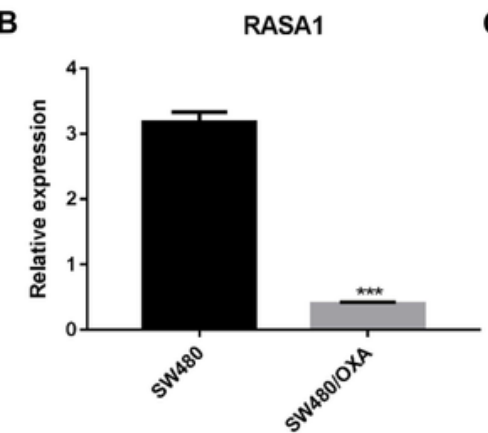

E

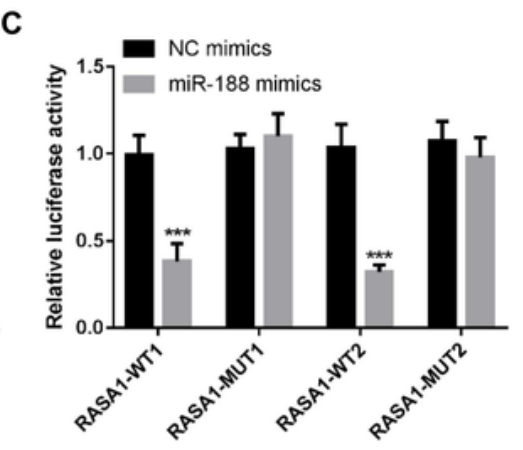

RASA1

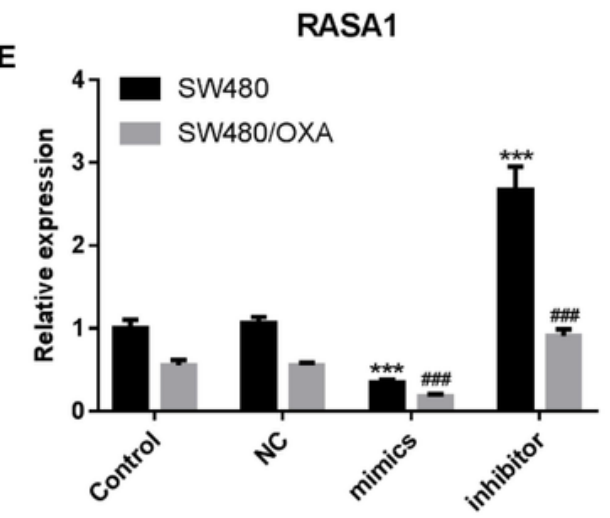

SW480/OXA 
collected and RNA was extract, the mRNA expression of miR-188 and RASA1 was detected by real-time PCR. ***P<0.001 as compared with SW480 NC group. \#\#\# P<0.001 as compared with SW480/OXA NC group. F. Cells were treated with mimics or inhibitor, the expression of RASA1 was determined by IF. The respective images were shown.

A

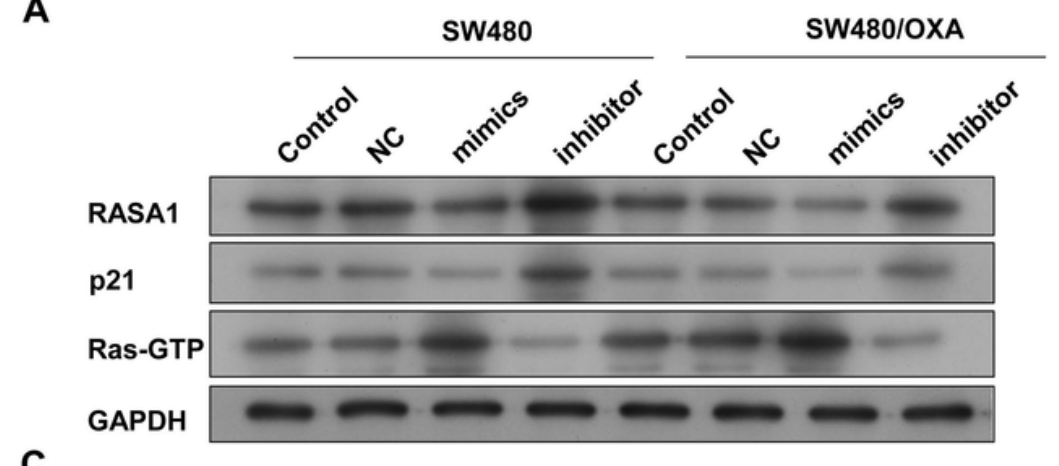

B

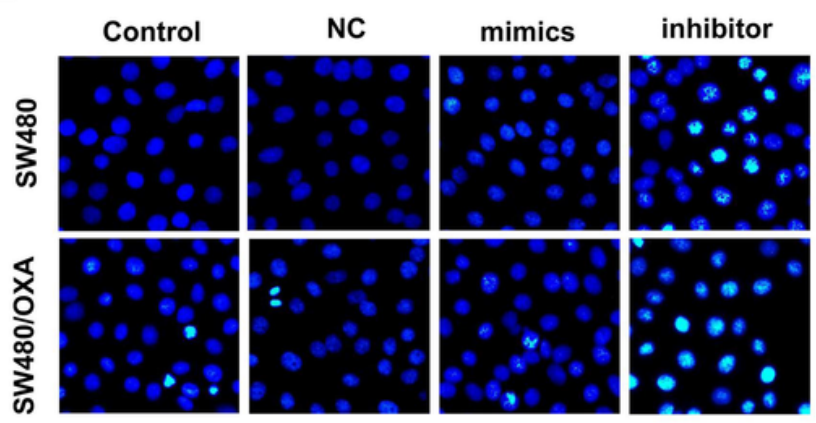

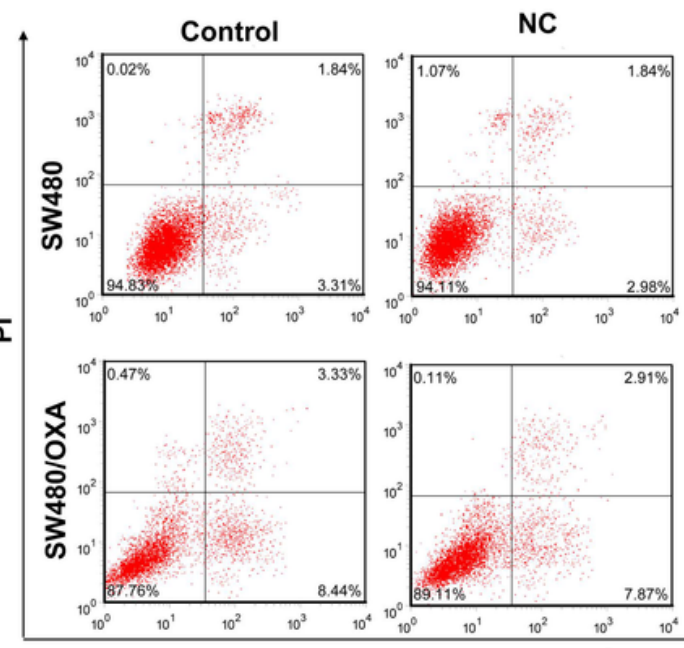
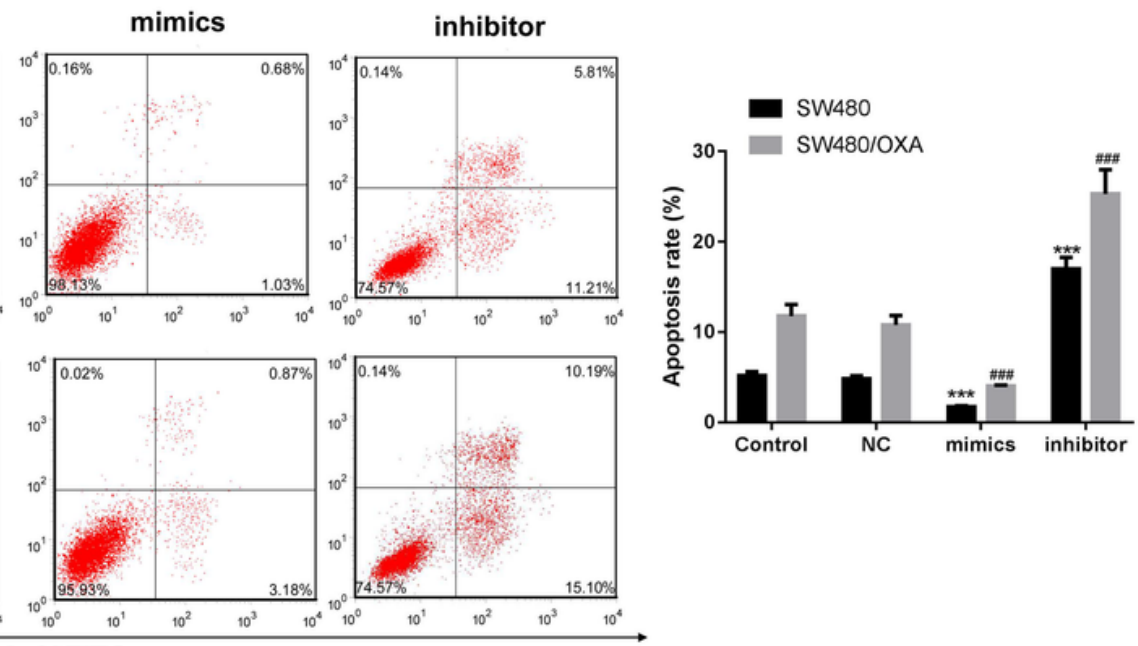

D
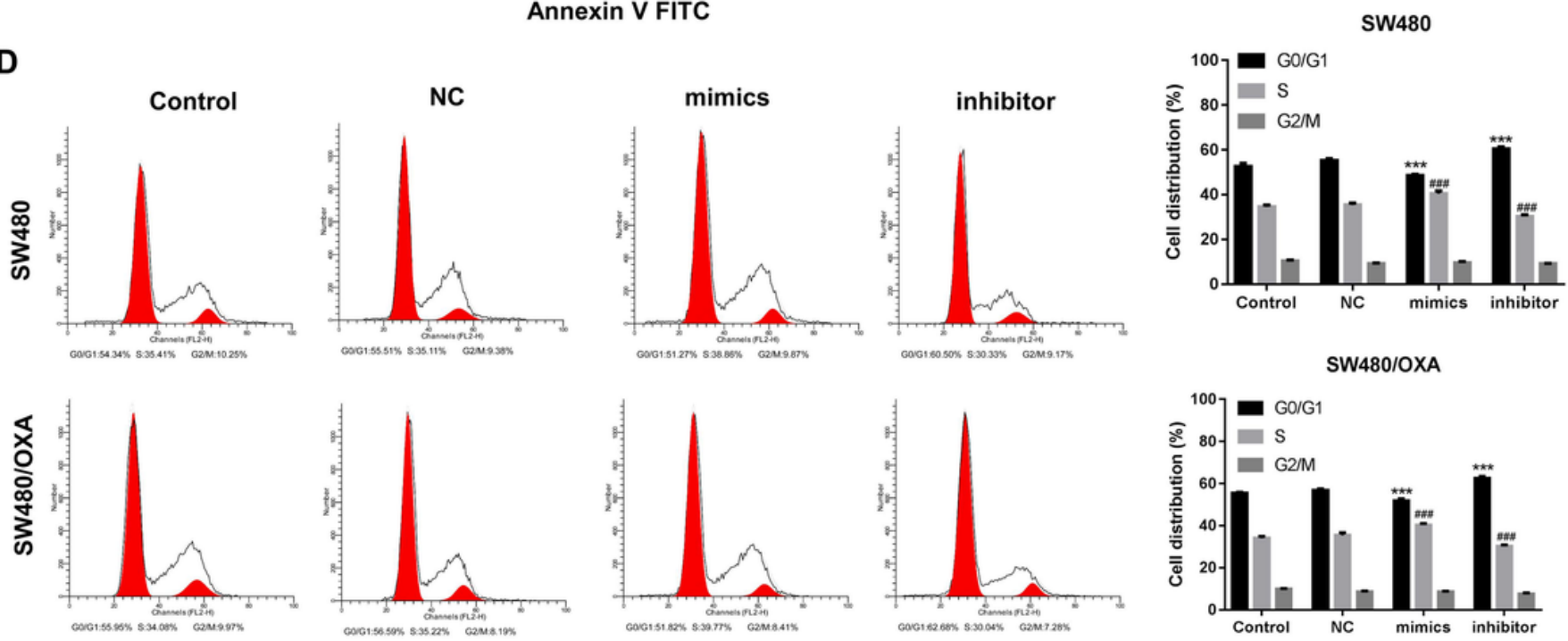

Figure 2

Suppression of miR-188 induces cell apoptosis in SW480/OXA cells A. SW480 or SW480/OXA cells were transfected with miR-188 mimics or inhibitor, after 48 hours, cells were lysed and Western blot was 
performed to detect the expression of RASA1, p21 and Ras-GTP. B. Cells were treated with miR-188 mimics or inhibitor, and stained with Hoechst 33342. The respective images were shown. C. Annexin V assay was performed and quantified after cells were transfected with miR-188 mimics or inhibitor $\mathrm{D}$. Cell cycle distribution was analyzed by flow cytometry. ${ }^{\star \star *} P<0.001$ as compared with SW480 NC group. $\# \# \# P<0.001$ as compared with SW480/OXA NC group.

A

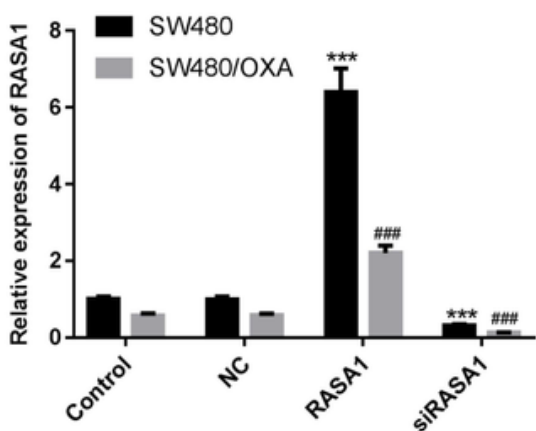

C

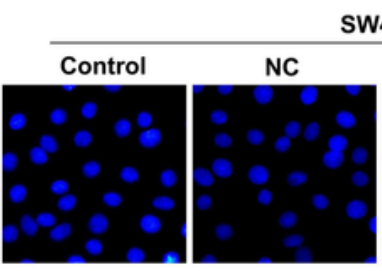

SW480

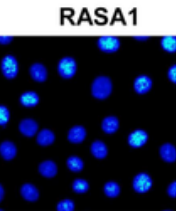

D
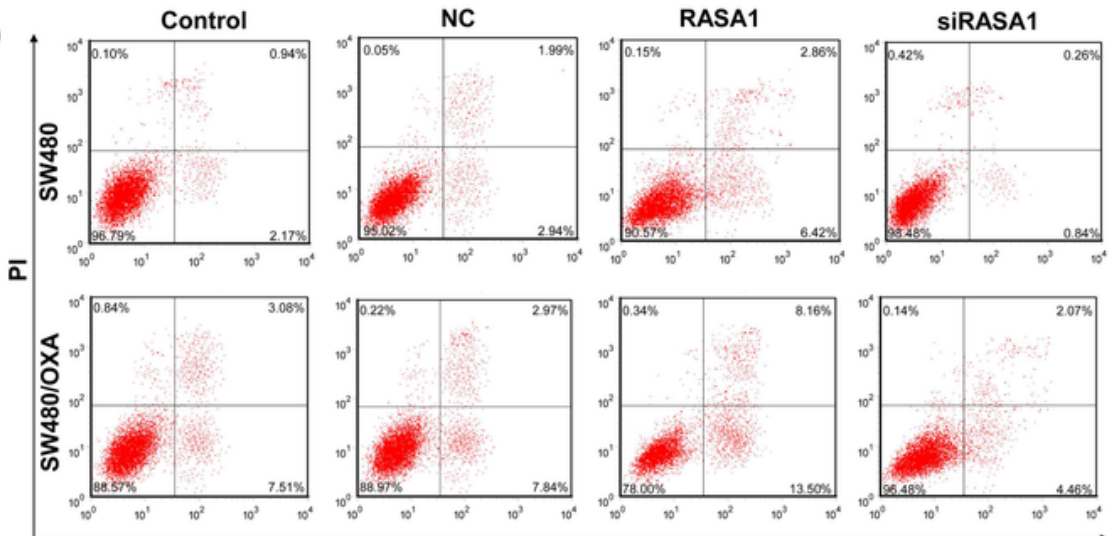

Annexin V FITC

E
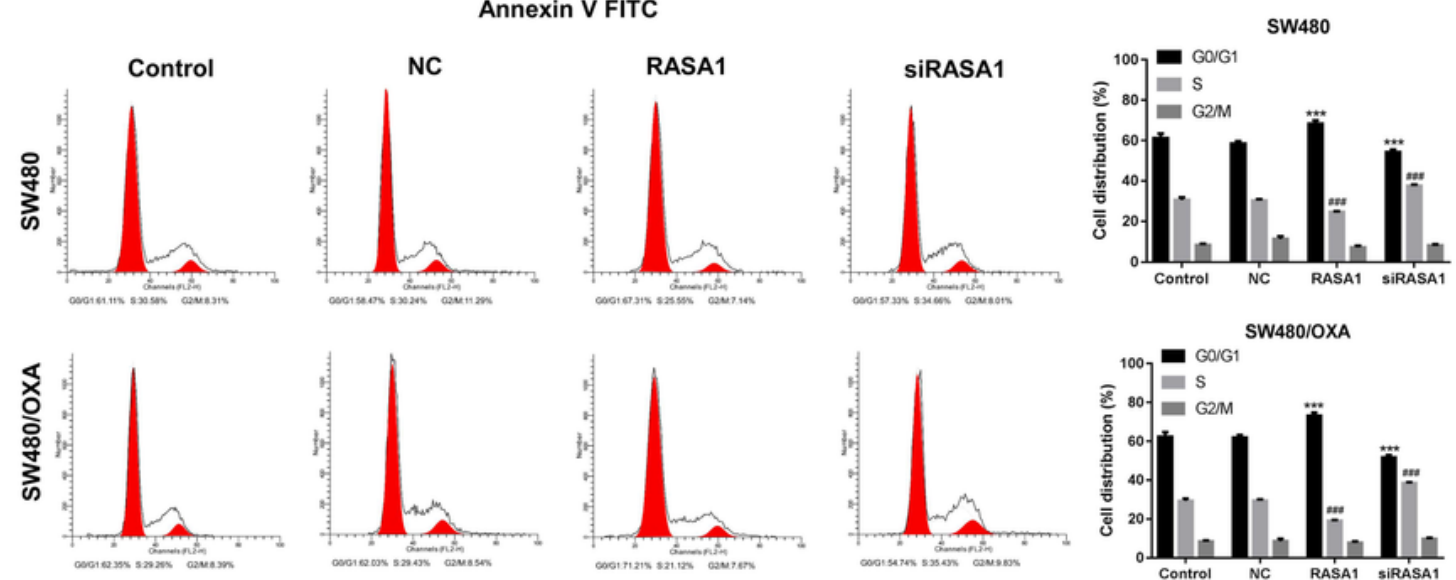

Figure 3 
Overexpression of RASA1 accelerates cell apoptosis and delays cell cycle. A. SW480 or SW480/OXA cells were transfected with RASA1 plasmid or RASA1 siRNA, after 48 hours, RNA was extracted and real-time PCR was performed. The relative mRNA expression of RASA1 was shown. B. Cells were treated as A, cells were lysed in lysis buffer and Western blot assay was performed to detect the expression of RASA1, p21 and Ras-GTP. C. Cells were stained with Hoechst 33342. The respective images were shown. D. Annexin V assay was performed and quantified to detect the apoptosis rate after cells were transfected with RASA1 overexpressed plasmid or RASA1 siRNA. E. Cell cycle distribution was analyzed by flow cytometry ***P<0.001 as compared with SW480 NC group. \#\#\#P<0.001 as compared with SW480/OXA NC group.
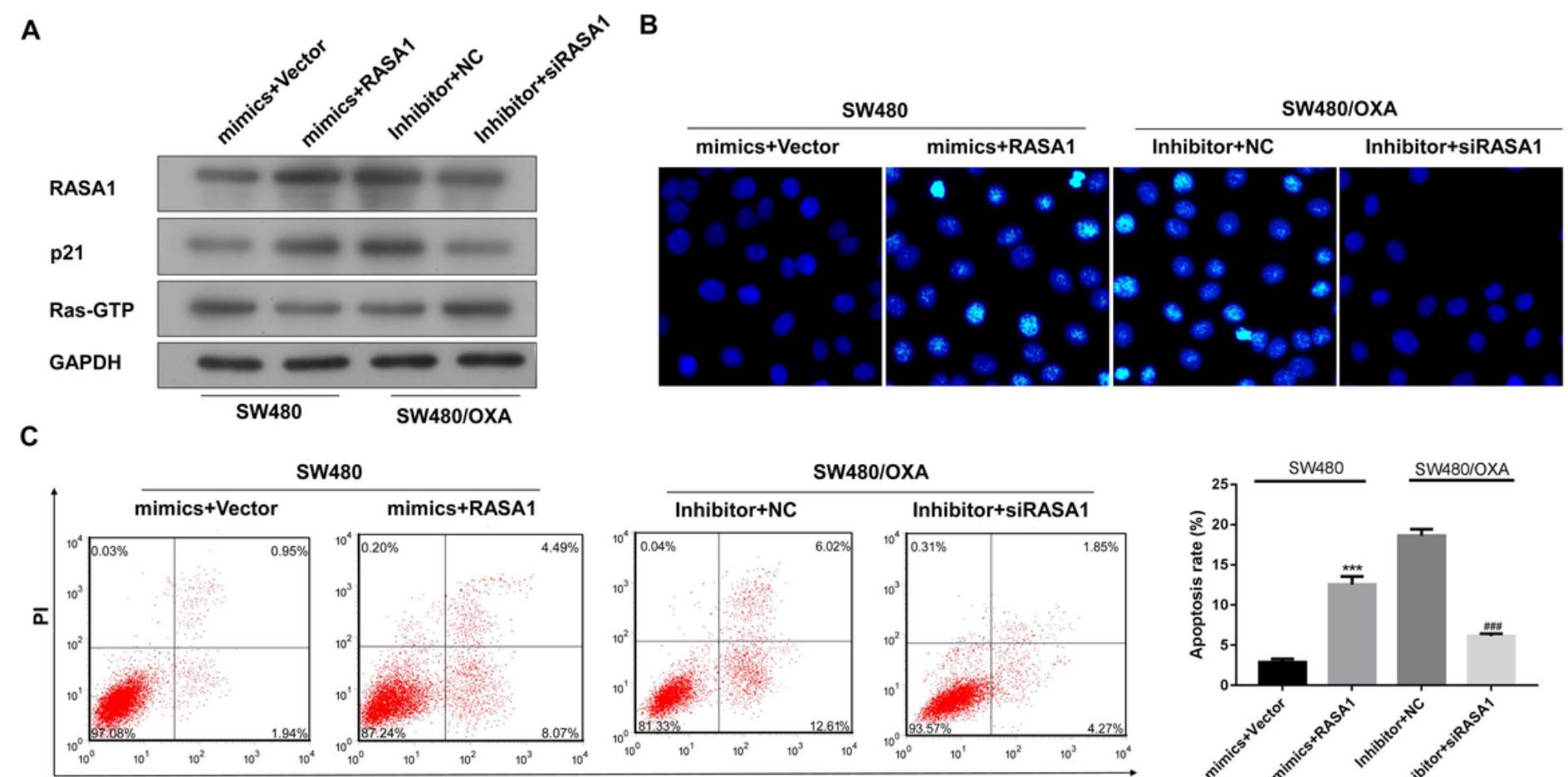

SW480
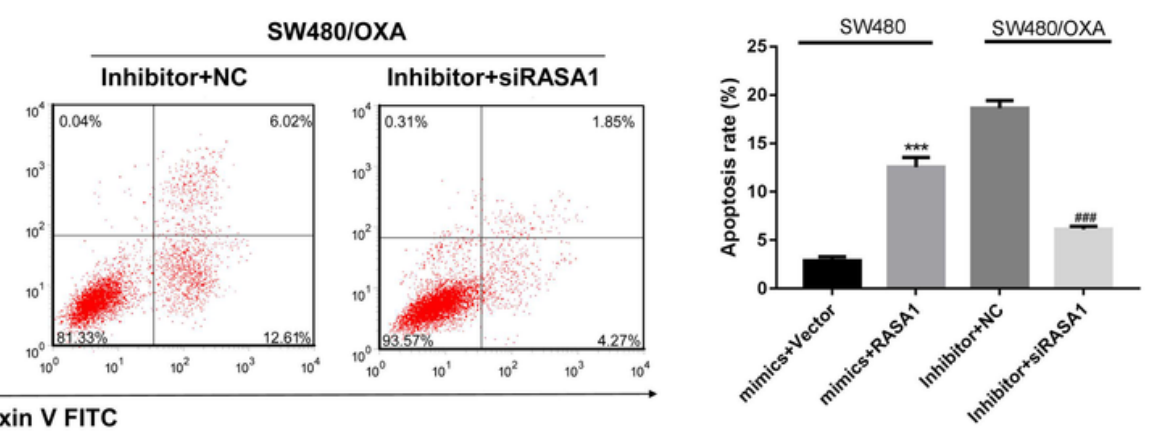

D

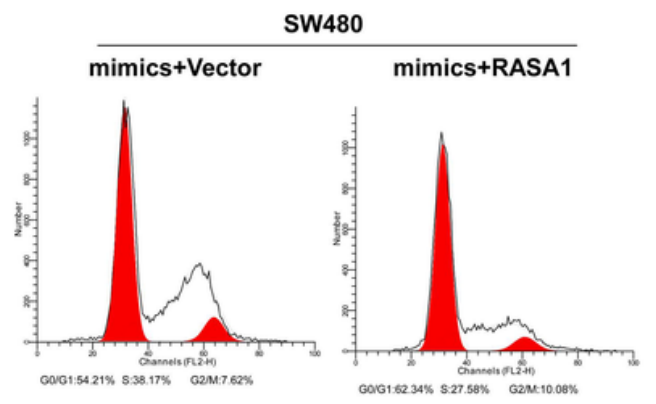

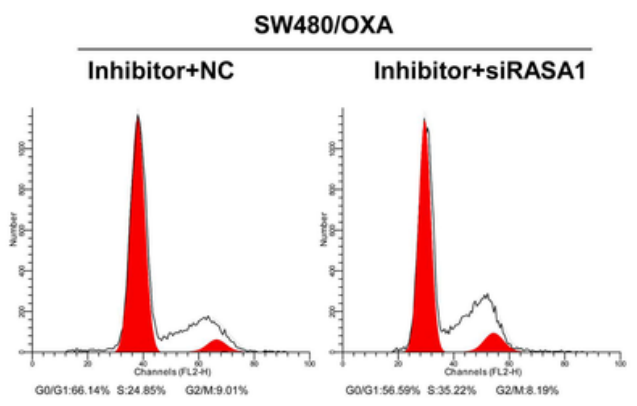

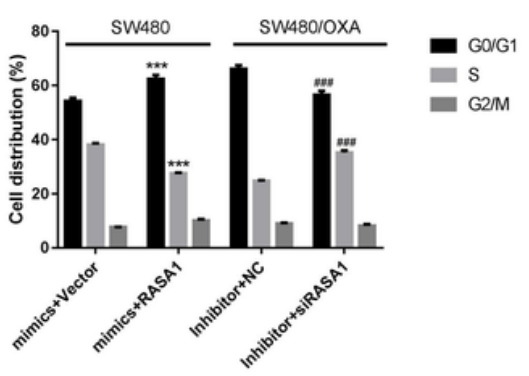

Figure 4

Suppression of miR-188 promotes SW480/OXA cell apoptosis via increasing RASA1 expression. A. SW480 or SW480/OXA cells were co-transfected with indicated siRNA or plasmid, after 48 hours, cells were lysed in lysis buffer and Western blot assay was performed to detect the expression of RASA1, p21 and Ras-GDP. B. Cells were stained with Hoechst 33342. The respective images were shown. C. Annexin V assay was performed and quantified after cells were co-transfected with indicated plasmid or siRNA. D. 
Cell cycle distribution was analyzed by flow cytometry $* \star \star P<0.001$ as compared with SW480mimics+vector group. \#\#\#P<0.001 as compared with SW480/OXA-inhibitor+siRNC group. 